\title{
FOURIER TRANSFORMS OF SURFACE-CARRIED \\ MEASURES AND DIFFERENTIABILITY OF SURFACE AVERAGES
}

\author{
BY WALTER LITTMAN ${ }^{1}$ \\ Communicated by Lawrence Markus, June 24, 1963
}

1. Introduction. We are given an $n$ surface $S$ (possibly with boundary) embedded in $R^{n+1}$ and a smooth mass density $\mu$ on the surface, vanishing near the boundary. We consider the following transformation of $C\left(R^{n+1}\right) \rightarrow C\left(R^{n+1}\right)$ :

$$
g(y)=\int_{x \in S} f(y-x) \mu(x) d S_{x} .
$$

Letting $D^{m}$ be a generic symbol for differentiation of order $m$, we ask the

Question: When does there exist an estimate of the type

$$
\left\|D^{m} g\right\|_{L_{p}} \leqq \text { constant } \cdot\|f\|_{L_{p}} ?
$$

This is related to the behavior at $\infty$ of the Fourier transform of the measure $\mu$. Our main result in that direction is the following:

ESTIMATE OF FOURIER TRANSFORMS. Let $S$ be a sufficiently smooth compact $n$-surface (possibly with boundary) embedded in $R^{n+1}, \mu$ a sufficiently smooth mass distribution on $S$ vanishing near the boundary of $S$. Suppose that at each point of $S, k$ of the $n$ principal curvatures are different from zero. Then

$$
I \equiv \int_{X \in S} e(X \cdot Y) \mu(X) d S_{X}=O\left(|Y|^{-k / 2}\right) .
$$

(Notation: $e(\cdot) \equiv e^{i(\cdot)}$ )

For the case of $\mu \equiv 1$ and surfaces of strictly positive Gaussian curvature this result has been proved by C. S. Herz [2] and previously by E. Hlawka [3]. Herz assumes $S$ to be of differentiability class $C^{[(n / 2)+2]}$. For simplicity we shall not keep track in this note of the smoothness assumptions on $S$ and $\mu$. The proof here, as it stands, does not give the best results in that direction. However, it can be modified (at the expense of making it somewhat more complicated) so as to get results reducing to those of [2] in the case of positive curvature.

${ }^{1}$ Preparation of this report was partially supported by the Office of Naval Research Contract Nonr 710 (54). 
To answer our "question," we notice that if the Fourier transform (3) is $O\left(|Y|^{-k / 2}\right.$ ) then (2) holds with $p=2$ and $m=k / 2$. Also, (2) holds with $m=0$ and $p=\infty$. By a theorem of A. P. Calderón [1] (2) holds with $m<k / p$ if $p \geqq 2$, and $m<k / p^{\prime}$ if $p \leqq 2$. We interpret fractional $n$ in the sense of [1].

2. Estimates for nonhorizontal part of surface. To estimate the integral $I$ in (3), first consider the case of curvature $\neq 0$. By rotation of axes take $Y=(0,0, \cdots, 0, y)$. The points $p_{1}, p_{2}, \cdots, p_{q}$ where $S$ is normal to the $X_{n+1}$ axis is finite in number. Decompose $\mu$ (by means of a partition of unity) into a sum $\mu=\mu_{0}+\mu_{1}+\cdots+\mu_{q}$, where $\mu_{0}$ vanishes near $p_{1}, \cdots, p_{q}$ and where the $\mu_{j}(j \neq 0)$ have disjoint supports containing the respective $p_{j}$. We first dispose of the integral $I_{2}$ involving $\mu_{0}$. We have

$$
I_{2}=\int e(z y) m_{z}^{1} d z
$$

where $m_{z}^{1}=(d / d z) m_{z}, S_{z} \equiv S \cap\left[x_{n+1} \leqq z\right]$, and $m_{z}=\int_{X \in S_{z}} \mu_{0}(X) d S_{X}$. To study $m_{z}$ further, decompose $\mu_{0} \equiv \mu_{01}+\mu_{02}+\cdots+\mu_{0 n}$ such that the support of $\mu_{0 k}(k \neq n+1)$ lies in a part of the surface $S$ with a representation (caret $\equiv$ omit)

$$
x_{k}=x_{k}\left(x_{1} \cdots \hat{x}_{k} \cdots x_{n+1}\right) .
$$

Then $m_{z}=\sum_{k=1}^{n} m_{z, k}$, where

$m_{z, k}=\int_{-\infty<x_{n+1} \leq z} \mu_{0 k} \frac{d S}{d x_{1} \cdots(d x)_{\hat{k}} \cdots d x_{n+1}} \cdot d x_{1} \cdots(d x)_{\hat{k}} \cdots d x_{n+1}$.

From this it follows that $m_{z, k}^{1}$ has compact support and has as many derivatives as $\mu_{0}$ or as the normal to $S$ has, whichever is less. Integrating by parts $\nu$ times in (4), we see that $I_{2}=O\left(|y|^{-\nu}\right)$, where $\nu$ is the differentiability class of $\mu$ or the normal to $S$, whichever is less.

3. Estimates near horizontal part of surface. To study the integral with $\mu_{j}, j>0$, simply write $\mu \equiv \mu_{j}$, and pick a coordinate system centered at point of tangency of the surface such that the tangent plane is given by $x_{n+1}=0$, the directions of principal curvature at the point of tangency are the $x_{j}$ axes $(j \neq n+1)$ and the part of the surface containing the support of $\dot{\mu}$ is given by $x_{n+1}=z\left(x_{1} \cdots x_{n}\right)$.

Since the first derivatives of $z$ vanish at the origin,

$$
z=\sum a_{i j} x_{i} x_{j}+R(x),
$$

where $R=O\left(|x|^{3}\right)$ and the $a_{i j}$ are constants and form a symmetric matrix with eigenvalues $\lambda_{1}, \lambda_{2}, \cdots, \lambda_{n}$ bounded away from zero. By 
a lemma of Morse [4] a new s-coordinate system can be introduced near the origin, also centered at the origin such that

$$
z\left(x_{1} \cdots x_{n}\right)=\sum \lambda_{j} s_{j}^{2} .
$$

The mapping $x \rightarrow s$ can be so chosen so that its differentiability class is two less than that of $z$. Furthermore, it is asymptotic to the identity as $x \rightarrow 0$, hence its Jacobian is close to one and the mapping is $1: 1$ for $x$ near 0 . Now the support of $\mu$ is picked so small that the Jacobian is greater than $\frac{1}{2}$.

Thus

$$
I=\int e\left(\sum \lambda_{j} s_{j}^{2} y\right) \phi(s) d s,
$$

where $\phi(s) \equiv \mu(x(s)) d S / d s$ and $d s=d s_{1} \cdots d s_{n}$.

Now introduce the $C^{\infty}$ function

$$
\begin{array}{ll}
\beta(t)=1 & \text { for }|t| \leqq \frac{a}{2}, \\
\beta(t)=0 & \text { for }|t| \geqq a
\end{array}
$$

and $\beta\left(s_{1}\right) \beta\left(s_{2}\right) \cdots \beta\left(s_{n}\right) \equiv B(s)$ and split $I$ of (5) into the sum $I=I_{3}+I_{4}$, where

$$
I_{4}=\int e\left(\sum \lambda_{j} s_{j}^{2} y\right) \phi(s) B(s) d s .
$$

Now $I_{3}$ is an integral of type $I_{2}$ already discussed.

Expanding $\phi$ by Taylor's formula with the remainder

$$
\begin{aligned}
{[\phi(s)=} & \left.\phi(0)+\sum_{1 \S|\alpha| \leqq m-1} b_{\alpha} s^{\alpha}+\sum_{|\alpha|=m} \tilde{\phi}_{\alpha}(s) s^{\alpha}\right], \\
I_{4}= & \phi(0) \prod_{j=1}^{n} \int e\left(\sum \lambda_{j} s_{j}^{2} y\right) \beta\left(s_{j}\right) d s_{j} \quad\left(\equiv I_{5}\right) \\
& +\sum_{1 \leqq|\alpha| \leqq m-1} b_{\alpha} \prod \int e\left(\lambda_{j} s_{j}^{2}\right) s_{j}^{\alpha_{j}} \beta\left(s_{j}\right) d s_{j} \quad\left(\equiv I_{6}\right) \\
& +\sum_{|\alpha|=m} \int e\left(\sum \lambda_{j} s_{j}^{2} y\right) \tilde{\phi}_{\alpha}(s) s^{\alpha} B(s) d x \quad\left(\equiv I_{7}\right) .
\end{aligned}
$$

Now 


$$
\int e\left(\lambda_{j} s_{j}^{2} y\right)\left(1-\beta\left(s_{j}\right)\right) d s_{j}=O\left(\frac{1}{\lambda_{j}|y|}\right)
$$

(let $s_{j}= \pm \sqrt{ } \eta$ and integrate by parts), hence

$$
I_{5}=\pi^{n / 2} \mu(0)|y|^{-n / 2} K^{-1 / 2}+O\left(|y|^{-n}\right)
$$

(where $K \equiv$ Gaussian curvature). Integrating the terms in $I_{1}$ by part an appropriate number of times shows that these terms are $O\left(|y|^{-n / 2-1 / 2}\right)$ or better. In $I_{7}$ the substitution $s_{j}= \pm \sqrt{ } \eta$ and integration by parts $[n / 2]+1$ times shows that $I_{7}=O\left(y^{-[n / 2]-1}\right)$ (we take $m>n / 2)$. Thus $I_{4}=O\left(y^{-n / 2}\right)$.

4. Zero Gaussian curvature. Finally, consider the case where only some of the principal curvatures are bounded away from zero. The set $A$ of points on $S$ at which $S$ is normal to the $x_{n+1}$ direction is no longer finite, but is compact. $A$ may be covered by a finite system of neighborhoods $N_{j}$ whose size can be chosen arbitrarily small. Introduce an appropriate partition of unity $\alpha_{0}, \alpha_{1}, \cdots, \alpha_{q}$ such that $\alpha_{0}$ has support in $S-U N_{j}, \alpha_{j}$ in $N_{j}(j \neq 0)$. The Fourier transform corresponding to $\alpha_{0}$ is of type $I_{2}$ already estimated. To estimate the remaining integrals, pick a point $p$ in $A$ and translate and rotate axes in $x_{1} \cdots x_{n}$ space so that the origin is at $p$ and the surface is described by

$$
x_{n+1}=z\left(x_{1}, \cdots, x_{n}\right)=\sum_{j=1}^{k} \lambda_{j} x_{j}^{2}+O\left(|x|^{3}\right) .
$$

Now consider the integral

$$
\int e\left(z\left(x_{1} \cdots x_{n}\right) y\right) \phi\left(x_{1} \cdots x_{n}\right) d x_{1} \cdots d x_{k},
$$

where $\phi=\mu(x) d S / d x_{1} \cdots d x_{n}$, and where the support of $\phi$ lies in the set

$$
\max _{1 \leqq j \leqq n}\left|x_{j}\right| \leqq a .
$$

For $x_{k+1}, \cdots, x_{n}$ fixed, the $k-1$ surface

$$
x_{n+1}=z\left(x_{1}, \cdots, x_{k}\right), \quad\left|x_{j}\right|<a, 1 \leqq j \leqq k
$$

in $x_{1}, x_{2}, \cdots, x_{k}, x_{n+1}$ space has Gaussian curvature bounded away from 0 , and this holds uniformly for $\left|x_{j}\right|<a, k+1 \leqq j \leqq n$, again provided $a$ is sufficiently small. Hence, from previous results,

$$
\int e(z(x) y) \phi\left(x_{1} \cdots x_{n}\right) d x_{1} \cdots d x_{k}=O\left(|y|^{-k / 2}\right)
$$


and this uniformly for $\left|x_{j}\right|<a, k+1 \leqq j \leqq n$. Hence

$$
\begin{aligned}
& \left|\int e(z(x) y) \phi\left(x_{1} \cdots x_{n}\right) d x_{1} \cdots d x_{n}\right| \\
& \quad \leqq \int\left|\int e(z(x) y) \phi(x) d x_{1} \cdots d x_{k}\right| d x_{k+1} \cdots d x_{n}=O\left(|y|^{-k / 2}\right),
\end{aligned}
$$

since the inner integral vanishes for $\max _{k+1 \leq j \leq n}\left|x_{j}\right| \leqq a$.

FinAL REMARK. That the estimate of the Fourier transform is uniform with respect to all directions of $Y$ follows from a careful analysis of the proof. Namely, every time some quantity is to be made "sufficiently small," it can be made so uniformly with respect to the direction of $Y$.

\section{BIBLIOGRAPHY}

1. A. P. Calderón, Lebesgue spaces, Proc. Sympos. Pure Math. Vol. 4, pp. 33-69, Amer. Math. Soc., Providence, R. I., 1961.

2. C. S. Herz, Fourier transforms related to convex sets, Ann. of Math. (2) 75 (1962), 215-254.

3. E. Hlawka, Integrale auf konvexen Körpern, Monatsh. Math. 54 (1950), 1-36.

4. M. Morse, The calculus of variations in the large, Amer. Math. Soc. Colloq. Publ. Vol. 18, Amer. Math. Soc., Providence, R. I., 1934.

UNIVERSITY OF MiNNESOTA 